\title{
Patterns of Gestational Weight Gain in Women with Overweight or Obesity and Risk of Large for Gestational Age
}

\author{
Wei Zheng a Wenyu Huang ${ }^{b} \quad$ Zhi Zhang $^{c} \quad$ Li Zhang $^{a} \quad$ Zhihong Tian $^{\text {a }}$ \\ Guanghui Lia Weiyuan Zhang ${ }^{a}$ \\ aDivision of Endocrinology and Metabolism, Department of Obstetrics, Beijing Obstetrics \\ and Gynecology Hospital, Capital Medical University, Beijing, China; ${ }^{b}$ Division of \\ Endocrinology, Metabolism and Molecular Medicine, Northwestern University Feinberg \\ School of Medicine, Chicago, IL, USA; 'Department of Obstetrics, Cangzhou People's \\ Hospital, Cangzhou, China
}

\section{Keywords}

Gestational weight gain - Latent class trajectory - Large for gestational age · Pregnancy · Birth weight

\begin{abstract}
Objective: Timing of gestational weight gain (GWG) may influence perinatal outcomes differently. This study aimed to find associations of latent GWG patterns with risk of large for gestational age (LGA) in women with overweight or obesity. Methods: A total of 4,438 women with overweight or obesity were included in the analyses. Latent trajectories of GWG associated with LGA were identified by trajectory analysis. GWG, risk of LGA and early pregnancy factors were compared between these identified groups. Results: This study identified four distinct GWG trajectory groups associated with LGA, each group including, respectively, 78.6, 19.0, 1.4, and $0.9 \%$ of the participants. Group 1 presented a typical curve with lower GWG in early pregnancy and relatively higher GWG in mid- and late pregnancy. Women in group 2 showed sustained high GWG and high LGA prevalence than women in group 1 (48.24 vs. $21.56 \%, p<0.0001)$. A catch-up in GWG after low weight gain did not result in significantly higher risk of LGA in group 3 compared to group 1. On the other hand, a rapid GWG in the first two-thirds of pregnancy followed by a strict weight control was associated with elevated risk of LGA in group 4 than group 1 (62.50 vs. 21.56\%, $p<0.0001)$. Conclusions: Women affected by overweight or obesity combined with high GWG in early mid-pregnancy were at elevated risk of LGA. Early clinical recognition of a poor GWG trajectory will enable early intervention in high-risk groups.


Zheng et al.: GWG Patterns in Women with Overweight or Obesity

\section{Introduction}

Obesity during pregnancy is a major public health concern because of its high prevalence and increased risk for maternal and fetal complications. Women with overweight or obesity are at higher risk of pregnancy complications including gestational diabetes (GDM), pregnancy-induced hypertension, caesarean section, and delivery of infants with high birth weight, which influence long-term cardiovascular health in both mothers and offspring [1-3]. Excessive gestational weight gain (GWG) is another growing health issue that has been drawing worldwide attention. It also contributes to the risk for adverse pregnancy outcomes mentioned above [3-9]. GWG is a marker of fetal growth and is directly associated with birth weight. Recent studies indicated that a majority of women affected by overweight or obesity gained more weight than the Institute of Medicine recommendations [10], and a joint effect of maternal obesity and excessive GWG on maternal and offspring outcomes was observed consistently $[3,10,11]$.

When assessing the effect of GWG on birth outcomes, researchers typically focus on total GWG. Nevertheless, GWG across specific intervals of pregnancy may contribute differently to perinatal outcomes. Some recent studies reassessed the association between GWG and pregnancy outcomes by subdividing the pregnancy period. However, conflicting findings were reported in this regard. Some studies reported that the majority of women with excessive total GWG gained more weight during early pregnancy [12]. Weight gain in early pregnancy is a determinant of infant birth weight and is associated with maternal and neonatal complications [12-15], while some studies declare that weight gain during mid- and late pregnancy is more important to infant birth weight since maternal GWG in these periods reflects growth of the fetus [16, 17]. A study by Karachaliou et al. [18] suggested that timing of GWG influences offspring outcomes differently. GWG in early pregnancy is associated with the risk of childhood obesity in offspring, while GWG in mid- and late pregnancy is associated with greater risk of delivery of infants born large for gestational age (LGA). However, it should be pointed out GWGs during different pregnancy periods are not independent factors. GWGs during mid- and late pregnancy might be affected by weight gain in previous intervals. Thus, constructing GWG trajectories and depicting its patterns helps to study GWG in a more comprehensive way. In this study, we constructed a latent class trajectory model to analyze the association of GWG trajectory patterns with neonatal birthweight in women with overweight or obesity, which is a high-risk group with high prevalence of inappropriate GWG requiring intensive intervention.

\section{Methods}

\section{Study Population}

The study population was from a large ongoing cohort study conducted at Beijing Obstetrics and Gynecology Hospital in China. The participants were selected from a total of 21,636 singleton pregnancy women who delivered between January 2014, and December 2017 in this hospital and participated in the cohort study. Singleton pregnancy women who were classified as overweight and obese according to the pre-pregnancy body mass index (BMI) by the Chinese guideline were included in the analysis [19]. Women with pre-existing chronic disease including type 2 diabetes, hypertension, liver, kidney, thyroid, or cardiovascular disease were excluded. The participants were enrolled in early pregnancy (6-8 weeks of gestation) and followed until delivery. This study has been approved by the Ethics Committee of Beijing Obstetrics and Gynecology Hospital. A written informed consent was obtained from all participants. 
Zheng et al.: GWG Patterns in Women with Overweight or Obesity

\section{Measurements}

Clinical information was collected through medical record review. Pre-pregnancy body weight was self-reported at first prenatal visit around 5-6th gestational week. Pre-pregnancy BMI was calculated as pre-pregnancy weight $/$ height ${ }^{2}\left(\mathrm{~kg} / \mathrm{m}^{2}\right)$. Women with overweight $(24 \leq$ BMI $<28$ ) and obesity (BMI $\geq 28 \mathrm{~kg} / \mathrm{m}^{2}$ ) were included in this study [19]. Total GWG and rate of GWG in early, mid-, and late pregnancy $(0-16,17-28$, and $\geq 29$ weeks' gestation) were calculated. Birth weight was classified as low birth weight (LBW) $(<2,500 \mathrm{~g})$, normal weight $(2,500-4,000 \mathrm{~g})$, and macrosomia ( $>4,000 \mathrm{~g})$. LGA (birthweights greater than the 90th percentile for gestational age) and SGA (birthweights below the 10th percentile for gestational age) were defined according to an international standard set by Villar et al. [20]. Less than 37 weeks' gestational age is defined as preterm. Early pregnancy metabolic measurements including fasting plasma glucose, total cholesterol (TC), triglycerides (TG), high-density lipoprotein-cholesterol (HDL-C), and low-density lipoprotein-cholesterol (LDL-C) were measured at 7-13 weeks of gestation. Impaired fasting glucose in early pregnancy was defined as fasting serum glucose level between 6.1 and $7.0 \mathrm{mmol} / \mathrm{L}$. GDM was diagnosed at 24-28 weeks of gestation using the American Diabetes Association (ADA) criteria [21].

\section{Statistical Methods}

We first conducted the multivariate logistic regression model to assess the differential impact of GWG timing on risk of LGA. The variables included in the model were selected by the stepwise method. Potential risk factors included maternal age, pre-pregnancy BMI, GWG in early, mid- and late pregnancy, parity, presence of GDM, TG, TC, HDL-C, and LDL-C during early pregnancy. Subsequently, we constructed a latent class trajectory model to explore GWG trajectories associated with LGA using PROC TRAJ in SAS [22, 23]. PROC TRAJ is a flexible semi-parametric mixture model to discover latent patterns of change over time and to estimate the corresponding probability of falling into each group. The optimum number of groups and dimensions was determined by Bayesian Information Criterion (BIC) values [24]. Quadratic functions were used to estimate the GWG trajectories. Risk of LGA between estimated patterns of GWG were compared by PROC TRAJ. Additionally, we classified the participants into four groups based on the latent trajectory models. We depicted trajectories of total GWG based on the grouping strategy using a generalized linear regression model. Comparisons of participant characteristics between group 1 and other groups were made using ANOVA followed by Dunnett's multiple comparison tests. We also depicted latent class trajectories for macrosomia, SGA, and LBW. All analyses were conducted using SAS 9.3.

\section{Results}

This study included a total of 4,438 women with overweight or obesity. Characteristics of the participants are presented in Table 1. The average pre-pregnancy BMI was 27.19 with a total GWG of $12.88 \mathrm{~kg}$. The proportions of having an infant born LGA and macrosomia in these women were notably high (24.48 and $11.71 \%)$, while proportions of having an infant born SGA and LBW were relatively low (1.99 and 4.21\%). We found that GWG throughout the pregnancy period (i.e., GWG in early, mid-and late pregnancy) is associated with LGA, adjusted for pre-pregnancy BMI, parity, presence of GDM, and HDL-C in early pregnancy (Table 2).

The results of the trajectory analysis are presented in Figure 1. This study identified four distinct GWG patterns associated with different risk of LGA, including 78.6, 19.0, 1.4, and $0.9 \%$ of the participants. Figure 1 estimated patterns of GWG rate in three gestational periods. Figure 2 described weight gain curves throughout pregnancy using the identified four groups. 
Table 1. Characteristic of the participants
Zheng et al.: GWG Patterns in Women with Overweight or Obesity

\begin{tabular}{lc}
\hline Participants, $n$ & 4,438 \\
Age, years & $31.66 \pm 3.86$ \\
Gravidity & $1.96 \pm 1.11$ \\
Parity & $1.28 \pm 0.48$ \\
History of EPL & $642(14.64)$ \\
Education level, high school or lower & $463(10.52)$ \\
Height, cm & $162.76 \pm 4.97$ \\
Pre-pregnancy weight, kg & $72.12 \pm 9.48$ \\
Pre-pregnancy BMI & $27.19 \pm 2.99$ \\
Early pregnancy metabolic measurements & \\
$\quad$ FPG, mmol/L & $5.00 \pm 1.00$ \\
$\quad$ TG, mmol/L & $1.41 \pm 0.77$ \\
$\quad$ TC, mmol/L & $4.50 \pm 0.77$ \\
$\quad$ HDL-C, mmol/L & $1.63 \pm 0.43$ \\
$\quad$ LDL-C, mmol/L & $2.14 \pm 0.64$ \\
GWG & \\
$\quad$ Total GWG, kg & $12.88 \pm 5.55$ \\
$\quad$ GWG in early pregnancy, kg & $1.64 \pm 3.19$ \\
$\quad$ GWG in mid-pregnancy, kg & $5.80 \pm 2.98$ \\
$\quad$ GWG in late pregnancy, kg & $5.17 \pm 3.06$ \\
\hline Pregnancy outcomes & \\
Gestational diabetes & $870(19.60)$ \\
Pregnancy-induced hypertension & $543(16.39)$ \\
Gestational age, weeks & $38.57 \pm 1.64$ \\
Caesarean section & $2,059(46.42)$ \\
LGeonatal birth weight, g & $3,439.2 \pm 529.3$ \\
SGA & $1,073(24.48)$ \\
Macrosomia & $820(11.99)$ \\
Low birth weight & $187(4.21)$ \\
Preterm & $295(6.65)$ \\
$\quad$
\end{tabular}

Data are presented as $n(\%)$ or mean \pm SD. EPL, early pregnancy loss; BMI, body mass index; FPG, fasting plasma glucose; TG, triglycerides; TC, total cholesterol; HDL-C, high-density lipoprotein cholesterol; LDL-C, low-density lipoprotein cholesterol; GWG, gestational weight gain; LGA, large for gestational age; SGA, small for gestational age.

Table 2. Association of GWG in different periods with LGA in women with overweight or obesity

\begin{tabular}{lcccc}
\hline & $\begin{array}{l}\text { Regression } \\
\text { coefficient }\end{array}$ & SE & $p$ value & OR (95\% CI) \\
\hline Pre-pregnancy BMI & 0.1145 & 0.0178 & $<0.0001$ & $1.12(1.08-1.16)$ \\
GWG in early pregnancy & 0.0649 & 0.0162 & $<0.0001$ & $1.07(1.03-1.10)$ \\
GWG in mid-pregnancy & 0.1074 & 0.0186 & $<0.0001$ & $1.11(1.07-1.16)$ \\
GWG in late pregnancy & 0.0350 & 0.0175 & 0.045 & $1.04(1.00-1.07)$ \\
Parity & 0.4876 & 0.1057 & $<0.0001$ & $1.63(1.32-2.00)$ \\
GDM & 0.2124 & 0.0602 & 0.0004 & $1.53(1.21-1.94)$ \\
HDL-C & -0.2940 & 0.1267 & 0.0203 & $0.75(0.58-0.96)$ \\
Constant & -5.2711 & 0.6153 & $<0.0001$ & \\
\hline
\end{tabular}

$p$ value and OR were calculated according to the multivariate logistic model by stepwise regression. GWG, gestational weight gain; LGA, large for gestational age; BMI, body mass index; GDM, gestational diabetes; HDL-C, high-density lipoprotein cholesterol. 
Fig. 1. Patterns of gestational weight gain rate in women with overweight or obesity by latent class trajectory modeling.
Zheng et al.: GWG Patterns in Women with Overweight or Obesity
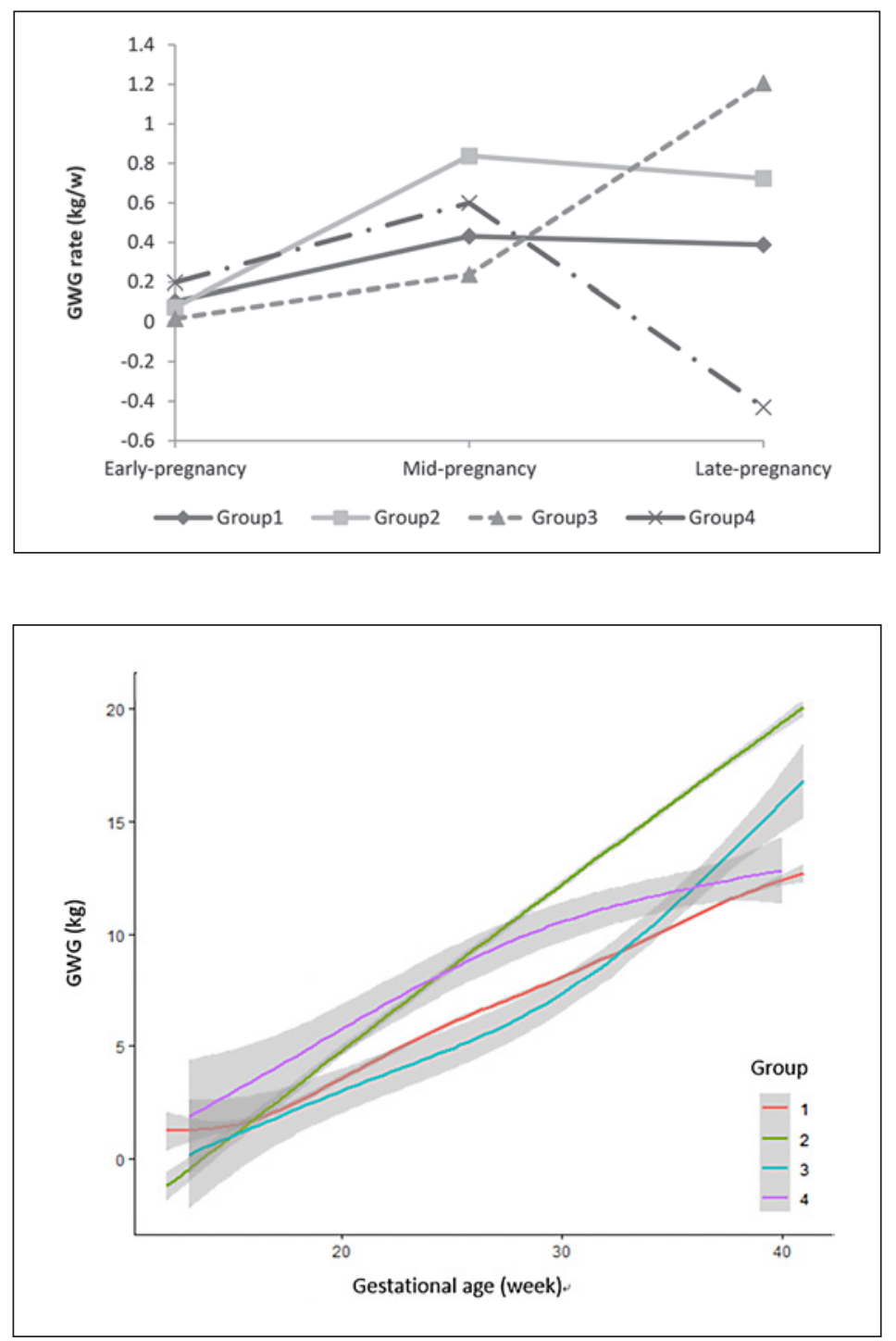

Fig. 2. Patterns of gestational weight gain in women with overweight or obesity throughout pregnancy.
Group 1 contained the majority of women. This group showed a lower GWG (1.69 kg) at 0-16 weeks of gestation and relatively higher GWG per week in mid- and late pregnancy (5.17 and $4.66 \mathrm{~kg}$ ) (Table 3). According to results from Figure 2, women in group 1 did not gain weight in the first 13 weeks of gestation, and then their body weight increased approximately linearly. Group 2 represented a subgroup of women with sustained higher GWG from mid-pregnancy and thereby caused an increase in LGA risk compared to group 1 . Women in this group had lower pre-pregnancy BMI and lower blood lipid levels in early pregnancy than group 1. Group 3 and group 4 contained a minority of women. Women in group 3 showed higher pre-pregnancy BMI, lower GWG in first two-thirds of the gestation and a catch-up in GWG in late pregnancy compared to group 1 . The high GWG in late pregnancy resulted in high total GWG but not increased LGA risk (Table 3). Women in group 4 showed higher GWG in early and midpregnancy and decreased GWG in late pregnancy compared with group 1. Although a significantly low total GWG was observed in group 4, the prevalence of LGA was remarkably high in this group. GWG trajectory pattern-associated risks of macrosomia and LGA were similar. On the other hand, no significant latent trajectory groups were shown to be associated with SGA or LBW. 
Zheng et al:: GWG Patterns in Women with Overweight or Obesity

Table 3. Comparison of GWG and early pregnancy factors between women with different GWG patterns

\begin{tabular}{lcccc}
\hline & Group 1 & Group 2 & Group 3 & Group 4 \\
\hline LGA & $839(21.56)$ & $205(48.24)^{*}$ & $14(33.33)$ & $15(62.50)^{*}$ \\
Pre-pregnancy BMI & $27.23 \pm 3.00$ & $26.47 \pm 2.47^{*}$ & $28.97 \pm 4.83^{*}$ & $28.22 \pm 4.23$ \\
Total GWG & $12.22 \pm 5.09$ & $19.45 \pm 4.54^{*}$ & $18.74 \pm 5.19^{*}$ & $5.91 \pm 5.99^{*}$ \\
GWG in early pregnancy & $1.69 \pm 3.11$ & $1.35 \pm 3.63$ & $0.79 \pm 4.22$ & $3.17 \pm 3.02^{*}$ \\
GWG in mid-pregnancy & $5.17 \pm 2.44$ & $9.98 \pm 2.31^{*}$ & $2.85 \pm 3.74^{*}$ & $7.19 \pm 4.13^{*}$ \\
GWG in late pregnancy & $4.66 \pm 2.41$ & $8.68 \pm 2.39^{*}$ & $14.29 \pm 2.04^{*}$ & $-4.91 \pm 1.73^{*}$ \\
FPG, mmol/L & $5.01 \pm 1.04$ & $4.90 \pm 0.67$ & $5.08 \pm 0.64$ & $4.80 \pm 0.35$ \\
TG, mmol/L & $1.42 \pm 0.77$ & $1.32 \pm 0.77^{*}$ & $1.22 \pm 0.73$ & $1.23 \pm 0.48$ \\
TC, mmol/L & $4.5 \pm 0.77$ & $4.48 \pm 0.84$ & $4.46 \pm 0.89$ & $4.83 \pm 0.65$ \\
HDL-C, mmol/L & $1.63 \pm 0.43$ & $1.70 \pm 0.41^{*}$ & $1.6 \pm 0.42$ & $1.79 \pm 0.41$ \\
LDL-C, mmol/L & $2.15 \pm 0.64$ & $2.05 \pm 0.66^{*}$ & $2.05 \pm 0.69$ & $2.35 \pm 0.62$ \\
\hline
\end{tabular}

GWG, gestational weight gain; LGA, large for gestational age; BMI, body mass index; TG, triglycerides; TC, total cholesterol; HDL-C, high-density lipoprotein cholesterol; LDL-C, low-density lipoprotein cholesterol. * Significant difference compared to group 1 by multiple comparison.

\section{Discussion}

This is the first study estimating GWG patterns with a latent class trajectory model in women with overweight or obesity. Overall, we identified four distinct GWG patterns associated with different risk of LGA and macrosomia. GWG in early, mid- and late pregnancy were all associated with elevated risk of LGA.

Group 1 showed a typical GWG trajectory with lower GWG in early pregnancy and relatively higher GWG in mid- and late pregnancy in overweight and obese women. GWG in group 1 was $1.69,5.17$, and $4.66 \mathrm{~kg}$ at $0-16,17-28$, and $29-40$ weeks of gestation, respectively. Sustained high GWG during pregnancy in group 2 resulted in higher total GWG and a significant higher prevalence of LGA. This result was consistent with previous findings regarding the effect of total GWG on neonatal birth weight $[4,25]$. There were other two special patterns discovered by latent class trajectory modeling. Interestingly, women in group 3 had higher total GWG but not significantly higher risk of LGA. In contrast, women in group 4 showed lower total GWG but remarkably higher risk of LGA than women in group 1 . These seemingly contradictory results may be due to the timing of GWG. In pattern 3, women gained less weight in the first two-thirds of the gestation and showed a catch-up in GWG in late pregnancy. Although this catch-up ended up with a significantly higher total GWG compared to GWG in women in group 1, it did not affect the LGA prevalence. This result might suggest that high GWG in late pregnancy alone would not increase risk of LGA, which is in agreement with a previous report by Gaillard et al. [17]. They found that higher weight gain in early pregnancy but not mid- or late pregnancy was associated with adverse cardiometabolic profiles in offspring. In group 4, women gained more weight in the first two-thirds of gestation but showed a decline in weight gain in the last period. Although women in this group did not have high pre-pregnancy BMI, high GWG, or other LGA-related factors, the prevalence of having a baby with LGA was higher than in women in group 1 . This result suggested the importance of GWG in early and mid-pregnancy which may influence risk of LGA. Studies by Davenport et al. [15], Walter et al. [26], and Rifas-Shiman et al. [27] also indicated the effect of early- and mid-pregnancy GWG on maternal and offspring outcomes.

Recent studies have been focusing on identifying critical periods for GWG with respect to managing GWG and priming maternal and offspring outcomes. However, only a few studies explored GWG trajectories. A study by Pugh et al. [28] estimated GWG trajectories in a normal- 
weight subgroup of US women. They discovered four groups with low, moderate, moderatehigh, and high GWG. In this study, we focused on the rate of GWG instead of total GWG. Therefore, we identified patterns varied in rates of GWG. This strategy enabled us to identify women with special type of GWG, such as weight control after a rapid weight gain, or a catch-up in GWG after low weight gain, thereby to discover critical periods of weight management during pregnancy to prevent LGA. A study by Catov et al. [29] also constructed GWG patterns in women with overweight or obesity by examining all combinations of weight gain groups in different gestational periods. They found that high GWG before 20 weeks, regardless of later weight gain, was related to LGA delivery. This finding is consistent with our findings. One possible explanation for this finding is that GWG in early pregnancy is largely contributed by maternal fat deposition, which is associated with higher placental transfer of nutrients [17] and may result in higher neonatal birthweight. However, further studies are needed to clarify these underlying mechanisms.

This study has certain limitations. First, exploring GWG trajectories using the latent class trajectory model has certain drawbacks, i.e. some types of GWG patterns might not be identified with a small number of participants. Due to the limited number of participants in identified subgroups and low prevalence of pregnancy complications, no GWG patterns associated with SGA or LBW were identified. Second, we could not analyze GWG in overweight and obese women separately due to a limited number of obese women. Therefore, we could only provide optimal GWG for the whole group. Further large-scale studies are needed to provide more information on GWG in overweight and obese women. Additionally, body weight at 12 or 13 weeks is not available in the majority of the participants, so we used weight at 16 weeks to calculate the rate of GWG in early pregnancy instead. A study by Hutcheon et al. [30] revealed that the rate of GWG was minimal until 15-20 weeks and then increased in a linear manner until term in women with overweight or obesity. Therefore, lacking data before 15 weeks of gestational age may not influence assessment of the GWG rate in the early period of pregnancy.

In summary, this study identified four distinct GWG trajectories associated with LGA. Sustained high GWG and a rapid GWG in early and mid-pregnancy followed by a strict weight control were associated with elevated risk of LGA. On the other hand, a catch-up in GWG after low weight gain did not result in significant higher risk of LGA. This is the first time women with catch-up GWG or weight control after a rapid GWG were identified to have different risk of LGA by latent class trajectory analyses. Early clinical recognition of a poor GWG trajectory may help to detect high-risk groups and conduct individualized intervention to minimize adverse outcomes. However, large-scale studies are needed to confirm these findings.

\section{Acknowledgement}

We thank the participants for their participation and medical staff for their work on information collection.

\section{Statement of Ethics}

This study was approved by the Ethics Committee of Beijing Obstetrics and Gynecology Hospital. Written informed consent was obtained from all participants.

\section{Disclosure Statement}

The authors declare no conflict of interest. 
Zheng et al.: GWG Patterns in Women with Overweight or Obesity

\section{Funding Sources}

This study was funded by Beijing Natural Science Foundation (S160001), National Natural Science Foundation of China (81671477), National key research and development program (2016YFC1000304), and Capital's Funds for Health Improvement and Research (CFH 2018-2-2112).

\section{Author Contributions}

W.Z. conducted the population study, analyzed and interpreted the data, and drafted the manuscript. W.H. participated in data analysis, interpretation, and draft revision. Z.Z. participated in data collection and results interpretation. L.Z. and Z.T. assisted with data collection and analysis. G.L. and Wy.Z. designed the study and critically revised the manuscript. All authors read and approved the final manuscript.

\section{References}

1 Rasmussen T, Stene LC, Samuelsen SO, Cinek O, Wetlesen T, Torjesen PA, et al. Maternal BMI before pregnancy, maternal weight gain during pregnancy, and risk of persistent positivity for multiple diabetes-associated autoantibodies in children with the high-risk HLA genotype: the MIDIA study. Diabetes Care 2009;32(10):1904-6.

2 Tsai IH, Chen CP, Sun FJ, Wu CH, Yeh SL. Associations of the pre-pregnancy body mass index and gestational weight gain with pregnancy outcomes in Taiwanese women. Asia Pac J Clin Nutr. 2012;21(1):82-7.

3 Kim SY, Sharma AJ, Sappenfield W, Wilson HG, Salihu HM. Association of maternal body mass index, excessive weight gain, and gestational diabetes mellitus with large-for-gestational-age births. Obstet Gynecol. 2014 Apr; 123(4):737-44.

4 Goldstein RF, Abell SK, Ranasinha S, Misso M, Boyle JA, Black MH, et al. Association of Gestational Weight Gain With Maternal and Infant Outcomes: A Systematic Review and Meta-analysis. JAMA. 2017 Jun;317(21):220725.

5 Gage SH, Lawlor DA, Tilling K, Fraser A. Associations of maternal weight gain in pregnancy with offspring cognition in childhood and adolescence: findings from the Avon Longitudinal Study of Parents and Children. Am J Epidemiol. 2013 Mar;177(5):402-10.

6 Fraser A, Tilling K, Macdonald-Wallis C, Hughes R, Sattar N, Nelson SM, et al. Associations of gestational weight gain with maternal body mass index, waist circumference, and blood pressure measured $16 \mathrm{y}$ after pregnancy: the Avon Longitudinal Study of Parents and Children (ALSPAC). Am J Clin Nutr. 2011 Jun;93(6):1285-92.

7 Nehring I, Schmoll S, Beyerlein A, Hauner H, von Kries R. Gestational weight gain and long-term postpartum weight retention: a meta-analysis. Am J Clin Nutr. 2011 Nov;94(5):1225-31.

8 Sridhar SB, Darbinian J, Ehrlich SF, Markman MA, Gunderson EP, Ferrara A, et al. Maternal gestational weight gain and offspring risk for childhood overweight or obesity. Am J Obstet Gynecol. 2014;211(3):259 e1-8.

9 Widen EM, Whyatt RM, Hoepner LA, Ramirez-Carvey J, Oberfield SE, Hassoun A, et al. Excessive gestational weight gain is associated with long-term body fat and weight retention at $7 \mathrm{y}$ postpartum in African American and Dominican mothers with underweight, normal, and overweight prepregnancy BMI. Am J Clin Nutr. 2015 Dec;102(6):1460-7.

10 Li C, Liu Y, Zhang W. Joint and Independent Associations of Gestational Weight Gain and Pre-Pregnancy Body Mass Index with Outcomes of Pregnancy in Chinese Women: A Retrospective Cohort Study. PLoS One. 2015 Aug;10(8):e0136850.

11 Kaar JL, Crume T, Brinton JT, Bischoff KJ, McDuffie R, Dabelea D. Maternal obesity, gestational weight gain, and offspring adiposity: the exploring perinatal outcomes among children study. J Pediatr. 2014 Sep;165(3):50915.

12 Carreno CA, Clifton RG, Hauth JC, Myatt L, Roberts JM, Spong CY, et al.; Eunice Kennedy Shriver National Institute of Child Health and Human Development (NICHD) Maternal-Fetal Medicine Units (MFMU) Network. Excessive early gestational weight gain and risk of gestational diabetes mellitus in nulliparous women. Obstet Gynecol. 2012 Jun;119(6):1227-33.

13 Retnakaran R, Wen SW, Tan H, Zhou S, Ye C, Shen M, et al. Association of Timing of Weight Gain in Pregnancy With Infant Birth Weight. JAMA Pediatr. 2018 Feb;172(2):136-42.

14 Cho EH, Hur J, Lee KJ. Early Gestational Weight Gain Rate and Adverse Pregnancy Outcomes in Korean Women. PLoS One. 2015 Oct;10(10):e0140376. 
Zheng et al.: GWG Patterns in Women with Overweight or Obesity

15 Davenport MH, Ruchat SM, Giroux I, Sopper MM, Mottola MF. Timing of excessive pregnancy-related weight gain and offspring adiposity at birth. Obstet Gynecol. 2013 Aug;122(2 Pt 1):255-61.

16 Durie DE, Thornburg LL, Glantz JC. Effect of second-trimester and third-trimester rate of gestational weight gain on maternal and neonatal outcomes. Obstet Gynecol. 2011 Sep;118(3):569-75.

17 Gaillard R, Steegers EA, Franco OH, Hofman A, Jaddoe VW. Maternal weight gain in different periods of pregnancy and childhood cardio-metabolic outcomes. The Generation R Study. Int J Obes. 2015 Apr;39(4):677-85.

18 Karachaliou M, Georgiou V, Roumeliotaki T, Chalkiadaki G, Daraki V, Koinaki S, et al. Association of trimesterspecific gestational weight gain with fetal growth, offspring obesity, and cardiometabolic traits in early childhood. Am J Obstet Gynecol. 2015 Apr;212(4):502.e1-14.

19 Department of Disease Control Ministry of Health, PR China. Guidelines for prevention and control of overweight and obesity in Chinese adults. Beijing: People's Medical Publishing House; 2006.

20 Villar J, Cheikh Ismail L, Victora CG, Ohuma EO, Bertino E, Altman DG, et al.; International Fetal and Newborn Growth Consortium for the 21st Century (INTERGROWTH-21st). International standards for newborn weight, length, and head circumference by gestational age and sex: the Newborn Cross-Sectional Study of the INTERGROWTH-21st Project. Lancet. 2014 Sep;384(9946):857-68.

21 American Diabetes Association. Standards of medical care in diabetes-2014. Diabetes Care. 2014 Jan;37 Suppl 1:S14-80.

22 Development G-bMo. Group-based Modeling of Development. Cambridge (Massachusetts): Harvard University Press; 2005.

23 Pugh SJ, Albert PS, Kim S, Grobman W, Hinkle SN, Newman RB, et al. Patterns of gestational weight gain and birthweight outcomes in the Eunice Kennedy Shriver National Institute of Child Health and Human Development Fetal Growth Studies-Singletons: a prospective study. Am J Obstet Gynecol. 2017 Sep;217(3):346. e1-11.

24 Jones BL, Nagin DS, Roeder K. A SAS procedure based on mixture models for estimating developmental trajectories. Sociol Methods Res. 2001;29(3):374-93.

25 Siega-Riz AM, Viswanathan M, Moos MK, Deierlein A, Mumford S, Knaack J, et al. A systematic review of outcomes of maternal weight gain according to the Institute of Medicine recommendations: birthweight, fetal growth, and postpartum weight retention. Am J Obstet Gynecol. 2009 Oct;201(4):339 e1-14.

26 Walter JR, Perng W, Kleinman KP, Rifas-Shiman SL, Rich-Edwards JW, Oken E. Associations of trimesterspecific gestational weight gain with maternal adiposity and systolic blood pressure at 3 and 7 years postpartum. Am J Obstet Gynecol. 2015 Apr;212(4):499.e1-12.

27 Rifas-Shiman SL, Fleisch A, Hivert MF, Mantzoros C, Gillman MW, Oken E. First and second trimester gestational weight gains are most strongly associated with cord blood levels of hormones at delivery important for glycemic control and somatic growth. Metabolism. 2017 Apr;69:112-9.

28 Pugh SJ, Albert PS, Kim S, et al. Patterns of gestational weight gain and birth weight outcomes in the NICHD Fetal Growth Study - Singletons: A prospective study. Am J Obstet Gynecol. 2017;217(3). https://doi. org/10.1016/j.ajog.2017.05.013.

29 Catov JM, Abatemarco D, Althouse A, Davis EM, Hubel C. Patterns of gestational weight gain related to fetal growth among women with overweight and obesity. Obesity (Silver Spring). 2015 May;23(5):1071-8.

30 Hutcheon JA, Platt RW, Abrams B, Himes KP, Simhan HN, Bodnar LM. Pregnancy weight gain charts for obese and overweight women. Obesity (Silver Spring). 2015 Mar;23(3):532-5. 